\title{
CONTINUUM EMISSION
}

"But if quasars had only been discovered in (say) 1973when we already knew more about lower-level activity in galactic nuclei (Seyferts etc), and when pulsars and X-ray binaries had convinced us that gravitational energy could be channelled efficiently into radiation - quasars would never have seemed 'sui generis', and theoretical ideas would have evolved less waywardly to their present state."

- Martin Rees (p.11) 
"The success of this program is a result of the dedication by a large number of collaborators who operate nine different telescopes that obtain data from $10^{8}-10^{18}$ $\mathrm{Hz}$ (Table I)."

- Joel Bregman (p.65) 
COMPACT CONTINUUM EMISSION FROM VIOLENTLY VARIABLE QUASARS AND BL LACERTAE OBJECTS

Joel N. Bregman

National Radio Astronomy Observatory

Edgemont Road

Charlottesville, VA 22903

USA

\begin{abstract}
A dozen BL Lacertae objects and violently variable quasars were observed repeatedly during the last six years by obtaining simultaneous measurements from the radio through $x$-ray region. The relationship between the radio-millimeter, infrared-ultraviolet, and $x$-ray regions are determined from single epoch spectra and variability measurements. The structure of the emitting regions and the enission mechanisms are discussed. The results of this progran are summarized.
\end{abstract}

\title{
1. INTRODUCTION
}

We report upon a program in which BL Lacertae objects and violently variable quasars are observed simultaneously over as much of the electromagnetic spectrum as possible. These observations are designed to shed light on the connection between various emitting regions, which are frequently studied separately (e.g. radio, optical, $X$-ray regions), and to determine the physical processes and conditions in the enitting plasma. Of a dozen sources, most have been observed at several epochs, providing important variability information. The success of this program is a result of the dedication by a large number of collaborators who operate nine different telescopes that obtain data from 108 - $1018 \mathrm{~Hz}$ (Table I).

\section{SINGLE EPOCH SPECTRA}

Multifrequency spectra have been obtained for the violently variable quasars $1156+295$ (Glassgold et al. 1983, wills et al. 1983), 3C 345 (Bregman et al. 1986), 3C 446 (Bregman et al. 1986) and the BL Lacertae objects IZw-187 (Bregman et al. 1982), 0735+178 (Bregman et al. 1984), OJ 287 (Pollock et al. 1985), 1413+135 (Bregman et 1. 1981), OJ 049, 0215+015, OV-236, BL Lac, 1308+326; 
TABLE I MULTIFREQUENCY COLLABORATORS

Dent, Balonek, Barvanis, O'Dea Aller, Aller, Hodge

Werner, Roellig

Harvey

Neugebauer, Solfer, Mathews, and Elias

Rieke, Lebofsky, Rudy, and Wisniewski

J.D. Bregman, Witteborn, Lester Impey, Willians, Brand

Hackwell

A. Smith, Pollock, Webb, Pica

Milier, Stephens

Wills, Wills

Bregman, Glassgold, Huggins, and Kinney

$\mathrm{Ku}$

Elvis, Schwartz, Tannanbaum

Pollock, Willmore

Mchardy

Maccagni
Univ. Mass
Univ. Mich.
NASA/AmeB
Univ. Texas
Palomar Obs.
and Caltech

Steward Obs.

NASA/AMES

UKIRT, REO

Univ. Wyoming

Univ. Florida

Lick Obs.

Univ. Texas

NYU and

NRAO

Columbia Univ.

CFA

U. Birmingham

U. Lelcester

CNR, Milan
1.5-90 GHz

4. 8-24 GHz

$1 \mathrm{~mm}-1 \mu n$

Far IR

$$
\begin{array}{r}
100-0.55 \mu \mathrm{m} \\
10-0.36 \mu \mathrm{m} \\
14-8 \mu \mathrm{m} \\
2.2-1.2 \mu \mathrm{m} \\
2.2-1.2 \mu \mathrm{m} \\
0.9-0.36 \mu \mathrm{m} \\
0.8-0.4 \mu \mathrm{m} \\
0.8-0.35 \mu \mathrm{m} \\
0.12-0.32 \mu \mathrm{m} \\
0.2-4 \mathrm{keV} \\
0.2-4 \mathrm{keV} \\
0.2-1 \mathrm{keV} \\
0.2-1 \mathrm{keV} \\
0.2-1 \mathrm{keV}
\end{array}
$$

and $1215+303$ (in preparation). The radio spectra of these sources, which are usually categorized as being "flat", sometimes show curvature and structure. The flatness of the spectrum appears to arise from several partially enitting regions of differing opacities; this interpretation is supported by VLBI observations (Cotton et al. 1980) and flux variability measurements (Aller et al. 1985). In nearly all of the sources we observed, the flat radio spectrum steepens in the 30 - $1000 \mathrm{GHz}$ region so that it has a slope of approximately -1. We interpret this turnover as the frequency at which all radio components become transparent. The $100-2 \mu m$ region is usually well described by a power law in the range -0.9 to -1.5 . In the optical and ultraviolet region, the spectrum sometimes steepens, reaching slopes of -3 occasionally. Some sources, such as IZw-187, have constant slopes from the infrared through the ultraviolet ( -0.9 in this case). The other extreme is represented by sources in with the spectrum steepens exponentially in less than a decade of frequency space. This feature, first noticed in red quasars (Rleke et al. 1977) and seen in sources such as $1413+135$, occurs at higher frequencies as well (in the ultraviolet in BL Lac, 3C 446), and is probably a common 
phenomena. In addition, when blue bumps have been detected, they occur only in violently variable quasars such as $3 C 345$ (the blue bump that was discussed for IZw-187 by Bregman et al. 1982 was not verified by more accurate observations).

There is sometimes a smooth connection between the IR-UV spectrum and the X-ray region, such as in IZw-187. However, for the majority of cases, an extrapolation of the IR-UV continuum to higher frequencies passes far below the $X$-ray flux density. This implies that $X$-rays emission arises either from a different physical process (thermal or inverse Compton emission) or from a different region, or both. The X-ray spectral slope of our sources, typically -1 , 18 generally steeper than the "universal" spectral slope of -0.62 (e.g. Rothschild 1983), although no single spectral slope characterizes quasars in the PalomarGreen survey (Elvis et al., this volume).

The radiated power in our sample is dominated by the contribution from the far infrared through optical

regions. This may be seen in sources such as $3 \mathrm{C} 345$, where the luminosity of the flat radio spectrum is comparable to that from the $\mathrm{X}$-ray region (if extrapolated to $165 \mathrm{keV}$ with a slope of -0.62 ) but only about a sixth of the luminosity from the far infrared - ultraviolet region. The luminosity in the blue bump is only about $0.65 \%$ of the total emitted power.

When a comparison is made between BL Lacertae objects and violently variable quasars, no obvious differences are apparent in the spectral shape save for the blue bump and the emission lines. One violently variable object, $3 C 446$, had been suggested to be an object intermediate between $B L$ Lacertae objects and violently variable quasars. It indeed has very small equivalent widths in the optical region, but the spectrum is also quite steep and the equivalent widths of the ultraviolet lines are more nearly like normal quasars. We find that for the number of ionizing photons emitted by $3 C 446$, its line strengths are perfectly normal (e.g. Its covering factor is like normal quasars). The ratio of the line strengths to the number of ionizing photons is also normal for the violently variable quasar 3C 345. Kinney et al. (1985) found that there was little dispersion in this ratio in normal quasars and argued that this implied that the ionizing radiation is not relativistically beamed into a small opening angle. By analogy, the ionizing radiation from the violently variable quasars $3 C 345$ and $3 C 446$ is also not beamed.

\section{VARIABILITY}

Each of the sources were selected because they have shown variability in the optical and radio wavebands. In 
3C 345 and OJ 287, there appears to be a connection in the variation in these two wavebands. In $3 C 345$, infrared and optical monitoring data reveal five outbursts during a 16 year period. There are also five outbursts defined by the $15 \mathrm{GHz}$ and $90 \mathrm{GHz}$ data. The radio outbursts are either coincident with or follow the IR-optical outbursts by $1-2$ years, and the $90 \mathrm{GHz}$ outbursts precede the $15 \mathrm{GHz}$ outbursts, typically by a year. The onset of outbursts as defined by the infrared or millimeter data coincide with the zero separation of individual superluminal components. One interpretation of these events is that the plasma responsible for optical outbursts moves outward, eventually becoming transparent at radio wavelengths and distinct as a VLBI component as it separates from the core.

The spectral variation in the IR-UV region during outbursts can differ from one object to another. During the dramatic outburst in $1156+295$, the spectrum retained its shape as it dimmed (over a period of about a week). However, a month later when the source was fainter, the spectrum was steeper and daily flux measurements at that time also showed no change in the spectral shape. Flux variation in which the spectral shape is preserved is quite common and has been seen in 0735+178, OJ 287, and other objects. In 3C 345, spectral variation is dramatic and during a particular infrared outburst, the $10 \mu \mathrm{m} f \mathrm{fux}$ changed less than at higher frequencies, so the spectrum became harder when the source brightened and softened when it faded. In the following year, a general dimming occurred that was barely perceptible at $100 \mu \mathrm{m}$ ( $8 x \mathrm{dimming}$ ) but became more dramatic at higher frequencies and decreased by a factor of 2.5 at $0.55 \mu \mathrm{m}$. This $f$ lux variation is most easily modeled by a hard and soft component, where the hard component appears and then fades.

The connection between $X$-ray variability and the enission at other wavelengths is especially complex. For IZw-187, the X-rays are probably just an extension of the IR-UV emission (the timescale and amplitude of variation is the same in all wavebands and the $X$-rays are smoothly connected to the IR-UV emission). However, in $0735+178$, the optical flux changed by $350 x$ while the $X$-ray emission was unchanged to within the uncertainties of the Einstein Observatory (30x; the $X$-rays may be consistent with the radio variation in which the fluxes changed by 5-40 $x$, depending upon the frequency). In 3C 345, the X-ray variation is not closely correlated with the IR-optical changes, but appears to be related to the radio flux variation. The sources $3 C 446$, and $O J 287$ show optical and $X-r a y$ flux variations that seem to occur at nearly the same time and either with similar amplitudes or with greater 
$X$-ray flux variation. However, $1156+295$ showed an $X-r a y$ outburst flanked by two optical outbursts, all of comparable amplitude (it 18 impossible to tell whether the X-ray outburst precedes or follows the optical outburst). In the handful of sources studies, the assortment of $X$-ray to optical behavior is astonishing. The results imply that $X$-rays are produced in several regions of (and) by several processes.

\section{MODELING}

The synchrotron-self-Compton model has frequently been applied to data such as ours. This model can be applied to our data when we assume (1) that the turnover in the millimeter and submillimeter region is the frequency at which the source becomes entirely transparent and (2) that the $X$-rays are produced through the inverse Compton process by the same electrons responsible for the submillimeter flux. The model results indicate that the plasma causing the submillineter emission has a magnetic field of 0.1 $100 \mathrm{G}, \mathrm{a}$ size of $1016-1017 \mathrm{~cm}$, and a density of 10 $104 \mathrm{~cm}-3$ (assuming a minimum electron energy of $15 \mathrm{MeV}$ ). Relativistic bulk motion is modest, with Lorentz factors of 1-3. These properties for the emitting plasma are significantly different from those found in the GHz region, where the sizes are greater by a hundredfold while the densities and magnetic fields are 102-105 times smaller. The variability data indicate that the optical, ultraviolet, and (sometimes) the $x$-ray emitting regions may be considerably smaller than the submillimeter region, with more intense magnetic fields imbedded in a denser plasma. Complex synchrotron-self-Compton models with special geometries, variation of the plasma parameters with radius and bulk acceleration of the plasma have been developed by a number of workers and provide a more realistic approach to Interpreting the observations (Blandford and Konigl 1979; Marscher 1980, 1983; Konigl 1981, Reynolds 1982a,b).

\section{REFERENCES}

Blandford, R.D., and Konigl, A. 1979, Ap.J., 232, 34. Bregman, J.N., Lebofeky, M.J., Aller, M.F., Rieke, G.H., Aller, H.D., Hodge, P.E., Glassgold, A.E., and Huggins, P.J. 1981, Nature, 293, 714.

Bregman, J.N., Glassgold, A.E., Huggins, P.J., Pollock, J.T., Pica, A.J., Smith, A.G., Webb, J.R., Ku, W.H.-M., Rudy, R.J., LeVan, P.D., Williams, P.M., Brand, P.W.J.L., Neugebauer, G., Balonek, T.J., Dent, W.A., Aller, H.D., Aller, M.F., Hodge, P.E. 1982, Ap.J., 253, 19.

Bregman, J.N., Glasegold, A.E., Huggins, P.J., Aller, H.D., Aller, M.F., Hodge, P.E., Rieke, G.H., Lebofsky, M.J., 
Pollock, J.T., Pica, A.J., Leacock, R.J., Smith, A.G., Webb, J., Balonek, T.J., Dent, W.A., O'Dea, C.P., Ku, W.H.-M., Schwartz, D.A., Miller, J.S., Rudy, R.J., and LeVan, P.D. 1984, Ap.J., 276, 454.

Cotton, W.D. et al. 1980, Ap.J. (Letters), 238, L123. Glassgold, A.E., Bregman, J.N., Huggins, P.J., Kinney, A.L., Pica, A.J., Pollock, J.T., Leacock, R.J.., Sinith, A.G., Webb, J.R., Wisniewski, W.Z., Jeske, N., Spinrad, H., Henry, R.B.C., Miller, J.S., Impey, C., Neugebauer, G., Aller, M.F., Aller, H.D., Hodge, P.E., Balonek, T.J., Dent, W.A., and O'Dea, C.P. 1983, Ap.J., 274, 101. Kinney, A.L., Huggins, P.J., Bregman, J.N., and Glassgold, A.E. 1985, Ap.J., 291, 128 .

Konigl, A. 1981, Ap.J., 243, 700.

Marscher, A.P. 1980, Ap.J., 235, 386. Marscher, A.P., 1983, Ap.J., 264, 296.

Pollock, A.M.T., Brand, P.W.J.L., Bregman, J.N., and

Robson, E.I. 1985, Apace Sclence Reviews, 40, 607.

Reynolds, S.P. 1982a, Ap.J., 256, 13.

Reynolds, S.P. 1982b, Ap.J., 256, 38 .

Rieke, G.H., Lebofsky, M.J., Kemp, J.C., Coyne, G.V., and

Tapia, S. 1977, Ap.J. (Letters), 218, L37.

Rothschild, R.E., Mushotzky, R.F., Baity, W.A., Gruber,

D.E., Matteson, J.L., and Peterson, L.E. 1983, AP.J., $269,423$.

Wills, B.J., Pollock, J.T., Aller, H.D., Aller, M.F., Balonek, T.J., Barvanis, R.E., Binzel, R.P., Chaffee, F.H., Dent, W.A., Douglas, J.N., Fanti, C., Garrett, D.B., Gregorini, L. Henry, R.B.C., Hill, R.E., Howard, R., Jeske, N., Kepler, S.O., Leacock, R.J., Mantovani, F., O'Dea, C.P., Padrielli, L., Perley, R., Pica, A.J., Puschell, J.J., Sanduleak, N., Shields, G.A., Snith, A.G., Thuan, T.X., Wade, C.M., Wasllewsk1, A.J., Webb, J.R., Wills, D., and Wisniewski, W.Z. 1983, Ap.J., 274, 62. 
Abramowicz : What is the relative number of objects which show quasi periodic variations to those which do not ?

Bregman : To answer this question, one would need a complete sample of high polarization sources. We have not observed a complete sample, but have selected sources that are known to have outbursts and that are bright enough to be observed with the IUE.

Cowsik : What are the reasons to believe that the continuum radiation is generated through Synchrotron and Compton processes?

Bregman : These sources are highly polarized in the $\mathbb{R}$-optical region and they vary rapidly, giving information on the size. As others have argued, these facts suggest synchrotron emission. There is little evidence that the $\mathrm{X}$-ray emission in inverse Compton radiation and this must be regarded as an assumption.

McAdam : Could you clarify the correlation between optical-infrared bursts and radio variability - In your presentation you said 4 or 5 outburst $\sim 1$ year earlier. Yet your abstract states .. "usually followed by radio outbursts ..." Is the correlation significant ?

Bregman : The outbursts are easy to identify but there is sometimes a problem in determining the beginning of an outburst when the sampling rate is too low. I encourage you to look at the data, which will appear in the Ap. J., Feb. 15, 1986. The connection between $\mathbb{R}$-optical and radio outbursts seems quite clear and we plan to examine this more quantitatively with Fourier techniques.

Cohen : In Neugebauer's $\mathbb{R}$ burst, a new hard component is needed because the radiative time scale is wrong for the existing soft component. In that case, how can the short time scale be explained for the hard component ?

Bregman : It is likely that the outburst occurs in a region much smaller than the size deduced from the multifrequency spectrum, which addresses the size of the emitting region where $\tau=1$ (in the millimeter region in 3C345). The decay of the hard component may be due to adiabatic expansion as the plasma flows into a larger region. 